\title{
Dynamic Power Coordination for Load Reduction in Dispatchable Wind Power Plants
}

\author{
Daria Madjidian*, Maxim Kristalny** and Anders Rantzer*
}

\begin{abstract}
In a dispatchable wind power plant, turbines are free to continuously vary their power production as long as the sum of their productions meets the total power demand. Previous research has shown that this freedom can be used to reduce structural loads by allowing turbines in the plant to coordinate their power. This paper explains the mechanisms that make power coordination useful for reducing structural loads on the turbine tower and the low speed shaft. In addition, it assesses the benefits of coordination at different operating points.
\end{abstract}

\section{INTRODUCTION}

In an attempt to accelerate investments in renewable energy, several regions around the world offer wind power plants (WPPs) feed-in tariffs in the form of guaranteed grid access and stable long term purchase agreements [1]. The effect of this extra-market treatment is that, unlike conventional generators that generate power to balance electrical load, WPPs lack an incentive to regulate their output power. Hence, additional amounts of reserve capacity need to be contracted to compensate for the inherent variability and uncertainty in the wind [2]. As the cost for these additional reserves will be higher at deeper penetration levels, it is likely that WPPs will be required to contribute more to the balancing effort in the future.

Such policies are emerging. For instance, several countries have updated their grid codes so that large WPPs are now required to respond to power requests from the system operator [3]-[5]. Moreover, in some countries, such as the United Kingdom and Spain, WPPs participate in electricity markets where they are penalized for deviations from contracted power levels [6], [7]. Also, several academic studies have investigated how WPPs can participate in electricity and ancillary markets. In [8], Kirby et al. analyze price differences between electricity and regulation markets in Texas and California during 2008 and 2009. Their results show that for a significant number of hours each year, regulation prices exceed electricity prices. This means that WPPs could benefit economically from curtailing power in order to provide grid support. In [9], the authors study optimal contract offers for a WPP participating in forward electricity markets. They show that, in order to avoid penalties on power deviations from contracted levels, the WPP often benefits from operating below maximum capacity.

Motivated by these developments, we consider a WPP, consisting of several wind turbines (WTs), scheduled to

\footnotetext{
*Department of Automatic Control, Lund Univesity, Sweden \{daria, rantzer\}@control.lth.se

**Department of Mechanical Engineering, Technion-IIT, Haifa, Israel kmaximetechunix.technion.ac.il
}

deliver a certain active power demand, which is lower than the WPP is capable of producing. This implies that there is freedom in distributing the power production among the WTs. In most work dedicated to dispatchable WPP control, this distribution is made with the sole purpose of attaining the power demand [10]-[12]. However, it is also possible to use the freedom in distributing power to improve additional aspects of WPP operation. For instance, in [13], this freedom is used to reduce active power losses in the transformers and lines inside the WPP. Another possibility, which is the topic of this paper, is to use the freedom in power distribution to reduce the structural loads experienced by the WTs. Instead of each WT following a fixed portion of the power demand, it can be allowed to continuously adjust its power production in response to local wind speed fluctuations. Since wind conditions are not uniform across the WPP, changes in power production that benefits one WT can be compensated for by WTs with opposite needs.

This idea, which we shall refer to as dynamic power coordination (DPC), was introduced in [14]. There, the problem is divided into two parts. First, optimal set points are computed offline for each WT using a receding horizon strategy. Then, the WTs are coordinated on-line to meet the total power demand. DPC subject to communication constraints was studied in [15] and [16]. Similar work was also presented in [17]. There, the problem is studied in a feedforward setting where only the wind speed is communicated between neighboring WTs.

The results in references [14]-[17], show that compared to situations where each WT follows a fixed portion of the power demand, DPC can result in a significant reduction in structural loads to both the tower and low speed shaft of the WTs. However, these references do not explain the mechanisms behind these load reductions. Moreover, they assume that the WTs are equipped with a pre-designed internal controller. The presence of internal controllers simplifies the coordination problem by reducing it to coordination of the power references. On the other hand, by limiting direct access to the pitch angle and the generator torque, the internal controller reduces the ability of the WT to respond to wind speed fluctuations. Hence, it limits the potential in coordinating the WTs.

In this paper, we study the benefits of DPC among WTs without an internal controller. While previous research was concerned with designing algorithms to carry out the coordination, we explain the mechanisms that makes DPC useful in terms of load reduction, and assess its benefits at different operating points. The remainder of this paper is 
organized as follows: In Section II, we introduce the WT model and explain the control objectives. In Section III, we study the benefits of allowing a WT to adjust its power production. DPC is studied in Section IV, and concluding remarks are presented in Section V.

\section{MODELING}

\section{A. Wind turbine model}

We adopt a model of the NREL 5 MW variable speed, collective pitch controlled WT based on [18]. The WT was introduced in [19] where it is described in detail. Note that parameter values that are not provided below can be found in [20]. A schematic overview of the WT is given in Figure 1. The aero-dynamics block describes the interaction with the

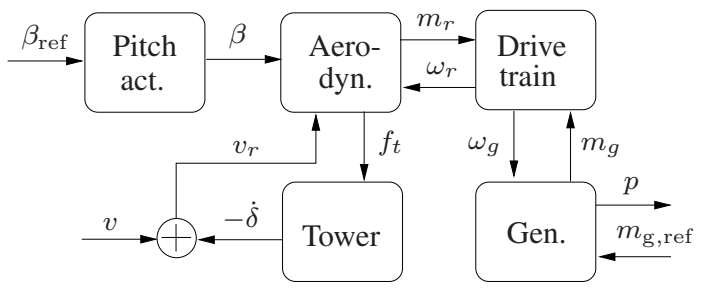

Fig. 1. Schematic overview of a variable speed pitch controlled WT.

wind,

$$
m_{r}=\frac{\rho}{2 \omega_{r}} \pi R^{2} C_{p}(\lambda, \beta) v_{r}^{3} \quad f_{t}=\frac{\rho}{2} \pi R^{2} C_{t}(\lambda, \beta) v_{r}^{2},
$$

where $m_{r}$ is the rotor torque, $\omega_{r}$ is the rotor speed, $f_{t}$ is the thrust force, and $v_{r}$ is the wind speed experienced by the rotor. The latter is given by $v_{r}=v-\dot{\delta}$, where $v$ is the ambient wind speed and $\delta$ is the fore-aft displacement of the WT nacelle. The parameter $\rho$ is the air density and $R$ is the rotor radius. The functions $C_{p}$ and $C_{t}$ are the power and thrust coefficient of the WT, respectively. They are static functions of the pitch angle, $\beta$, and the tip speed ratio, $\lambda=\frac{R \omega_{r}}{v_{r}}$.

The drive train connects the rotor and generator shafts via a gear box with a gear ratio of $n_{g}$. It is modeled as a third order system

$$
J_{r} \dot{\omega}_{r}=m_{r}-m_{\mathrm{sh}}, \quad J_{g} \dot{\omega}_{g}=\frac{m_{\mathrm{sh}}}{n_{g}}-m_{g}, \quad \dot{\theta}=\omega_{r}-\frac{\omega_{g}}{n_{g}},
$$

where $\omega_{g}$ is the generator speed, $\theta$ is the torsion of the low speed shaft and $m_{\mathrm{sh}}$ is the restoring shaft torque:

$$
m_{\mathrm{sh}}=b_{s} \dot{\theta}+k_{s} \theta \text {. }
$$

The parameters $J_{r}, J_{g}, b_{s}$ and $k_{s}$ are the rotor inertia, generator inertia, torsional damping and torsional stiffness, respectively. The drive train has a poorly damped resonant mode at $\omega_{\mathrm{sh}}=14 \mathrm{rad} / \mathrm{s}$.

The generator is modeled as a first order system with a time constant of $\tau_{g}=0.1 \mathrm{sec}$ :

$$
\dot{m}_{g}=\frac{1}{\tau_{g}}\left(m_{g, \text { ref }}-m_{g}\right) \quad p=m_{g} \omega_{g},
$$

where $p$ is the electrical power, and $m_{g}$ and $m_{g, \text { ref }}$ are the generator torque and its reference, respectively.
The tower block describes the fore-aft displacement of the nacelle, which is modeled as a spring mass system excited by the thrust force:

$$
m_{t} \ddot{\delta}=f_{t}-f_{\text {tow }}, \quad f_{\text {tow }}=b_{t} \dot{\delta}+k_{t} \delta,
$$

where $m_{t}, b_{t}$, and $k_{t}$ are mass, damping and stiffness parameters, respectively, and $f_{\text {tow }}$ is the restoring tower bending force. The tower has a resonance frequency of $\omega_{\text {tow }}=2$ $\mathrm{rad} / \mathrm{s}$.

The pitch actuator is modeled as a first order system $\dot{\beta}=$ $\frac{1}{0.3}\left(\beta_{\text {ref }}-\beta\right)$ where $\beta_{\text {ref }}$ is the pitch angle reference.

\section{B. Generalized control plant}

To facilitate analysis, we consider operation around an operating point, where the WT model can be approximated by an LTI plant. The operating point is determined by the mean wind speed, $v_{\text {nom }}$, and an external demand on power production, $p_{\text {nom. }}$. The choice of operating point will be explained in Section III. Unless otherwise stated, all signals will henceforth describe the deviation from their nominal value at the operating point.

For control purposes, we use the generalized plant $P$ depicted in Figure 2. It incorporates a model of the WT, the exogenous disturbances and the regulated outputs.

The inputs are the control signal $u=\left[\begin{array}{ll}\beta_{\text {ref }} & m_{g, \text { ref }}\end{array}\right]^{T}$ and the exogenous noise $w=\left[\begin{array}{ll}w_{v} & w_{e}\end{array}\right]^{T}$, where $w_{v}$ and $w_{e}$ are both Gaussian white noise processes with unit intensity and indepen-

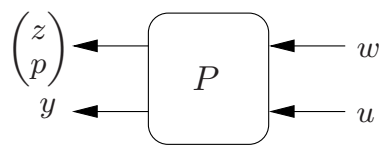

Fig. 2. Linear turbine model. dent of each other. The process $w_{v}$ generates the wind speed fluctuations according to $v=W_{v} w_{v}$, where the filter $W_{v}(s)=\frac{0.1065}{s+0.0143}$ was identified from real WT data in [21]. The process $w_{e}$ generates the measurement noise. We assume that we can only measure the generator speed. The measured signal is given by $y=\omega_{g}+W_{e} w_{e}$. To prevent the controller from exciting unmodeled resonant modes at higher frequencies (e.g. blade bending) we set $W_{e}=0.2 \frac{s}{s+\omega_{e}}$, where the corner frequency, $\omega_{e}=\frac{\pi}{5}$, is chosen to be ten times lower than the first edgewise blade resonance frequency [19]. The regulated signals are the power, $p$, and the vector

$$
z=\left(\begin{array}{lllll}
\omega_{r} & m_{\mathrm{sh}} & f_{\text {tow }} & \tilde{\beta} & \tilde{m}_{g}
\end{array}\right)^{T},
$$

whose elements relate to relevant mechanical loads and limitations. The rotor speed, $\omega_{r}$, should not exceed its rated value. The tower force variation, $f_{\text {tow }}$, needs to be kept small in order to reduce fatigue damage to the tower. Similarly, to reduce fatigue damage to the gear box and low speed shaft, we penalize the shaft torque $m_{\mathrm{sh}}$. The signals $\tilde{\beta}=$ $W_{\beta} \beta_{\text {ref }}$ and $\tilde{m}_{g}=W_{m} m_{g, \text { ref }}$ are related to the pitch activity and torque activity, respectively. In order to avoid damping tower and drive train oscillations through oscillations in the pitch angle and generator torque, we set $W_{\beta}(s)=$ $\frac{s^{2}}{s^{2}+0.2 \omega_{\text {tow }} s+\omega_{\text {tow }}^{2}}$ and $W_{m}(s)=\frac{s^{2}}{s^{2}+0.2 \omega_{\text {shaft }} s+\omega_{\text {shaft }}^{2}}$. 


\section{Wind power plant model}

Wind speed variations at WTs in a WPP are correlated. However, studies show that they are correlated only at low frequencies, which are less important when controlling WT dynamics in the vicinity of an operating point [21]. The larger the distance between turbines, the lower the frequencies where the WTs are coupled [22]. For the sake of simplicity, when considering several WTs around their operating points, we assume that the distance between them is large enough to neglect WT coupling.

\section{BENEFIT OF ALLOWING WT POWER VARIATION}

The purpose of this section is to investigate the potential for reducing fatigue loads by allowing WTs to vary their power production. To this end, we consider operation of a single WT under two different power tracking policies. Under Policy 1 , the variation in power production is tightly constrained, whereas under Policy 2, this constraint is removed. Controllers for each of these policies are given by the solutions to Problems 1 - 2 stated below. To shorten notation, for a zero mean stationary process $x$, we let $\|x\|^{2}$ denote its variance: $\|x\|^{2}=\mathbf{E} x^{2}(t)$. As a measure of tower loading, we consider the standard deviation of the tower force, $\left\|f_{\text {tow }}\right\|$. Similarly, the low speed shaft load is defined as $\left\|m_{\mathrm{sh}}\right\|$. Let $\bar{\omega}_{r}, \bar{\beta}, \bar{m}_{g}$ and $\bar{p}$ be positive scalars.

Problem 1 (Tight power tracking): Given $\eta \in[0,1]$, find a stabilizing controller, $K_{1}: y \rightarrow u$, that minimizes

$$
J\left(m_{\text {sh }}, f_{\text {tow }}\right)=\eta\left\|m_{\text {sh }}\right\|^{2}+(1-\eta)\left\|f_{\text {tow }}\right\|^{2}
$$

and satisfies

$$
\left\|\omega_{r}\right\| \leq \bar{\omega}_{r} \quad\|\tilde{\beta}\| \leq \bar{\beta} \quad\left\|\tilde{m}_{g}\right\| \leq \bar{m}_{g},
$$

as well as

$$
\|p\| \leq \bar{p}
$$

The constraints in (3) constitute predefined limits on the amount of rotor speed variation, pitch activity, and generator torque activity. The constraint (4) limits the amount of variation in the WT's power production.

Problem 2 (Relaxed power production): Given $\eta \in[0,1]$, find a stabilizing controller, $K_{2}: y \rightarrow u$, that minimizes (2) and satisfies (3).

Note that, as opposed to Problem 1, there is no constraint on power tracking in Problem 2.

Remark 1: Problems $1-2$ above are constrained LQGproblems. Their solution can be found by means of Lagrangian relaxation and subsequent iteration over the dual variables. For more information, we refer to [23] and [24], where it was shown that the duality gap is zero.

In order to set reasonable values on the bounds in (3)(4), we introduce a standard WT controller, denoted $K_{0}$, from [19]. Above rated wind speed, the controller keeps the WT within its mechanical and electrical limits. This is achieved by varying the pitch angle to maintain rated rotor speed and adjusting the generator torque to attain rated power. Below rated wind speed, $K_{0}$ tries to extract maximum power. This is done by fixing the pitch angle to the angle
TABLE I

PERFORMANCE OF DIFFERENT CONTROLLERS.

\begin{tabular}{c|cccccc} 
& $\begin{array}{c}\left\|w_{r}\right\| \\
\mathrm{mrad} / \mathrm{sec}\end{array}$ & $\begin{array}{c}\left\|m_{\mathrm{sh}}\right\| \\
\mathrm{kNm}\end{array}$ & $\begin{array}{c}\left\|f_{\text {tow }}\right\| \\
\mathrm{kN}\end{array}$ & $\begin{array}{c}\|\tilde{\beta}\| \\
-\end{array}$ & $\left\|\tilde{m}_{g}\right\|$ & $\begin{array}{c}\|p\| \\
\mathrm{kW}\end{array}$ \\
\hline$K_{0}^{9}$ & 83 & 383 & 75.8 & 0 & 66 & 619 \\
$K_{0}^{15}$ & 5.4 & 37.66 & 33.20 & 0.19 & 46 & 12 \\
\hline$K_{1}^{15}$ & 5.5 & 18.72 & 20.47 & 0.19 & 200 & 12 \\
$K_{2}^{15}$ & 5.4 & 147.89 & 11.68 & 0.19 & 200 & 204 \\
$K_{r}^{15}$ & 325.2 & 802.70 & 11.60 & 0.19 & 200 & 12 \\
$K_{p, r}^{15}$ & 55.2 & 14.55 & 11.59 & 0.19 & 200 & 175 \\
\hline$K_{1}^{9}$ & 34.4 & 50.00 & 39.57 & 0.19 & 200 & 12 \\
$K_{2}^{9}$ & 103.3 & 10.76 & 11.41 & 0.19 & 200 & 192 \\
$K_{3,1}^{9}$ & 80.1 & 50.00 & 28.20 & 0.19 & 200 & 136 \\
\hline \hline
\end{tabular}

that corresponds to the highest power capture and using the generator torque to track optimal rotor speed.

In the next two subsections, we will compare Policy 1 and Policy 2 controllers at nominal wind speeds of $15 \mathrm{~m} / \mathrm{s}$ and $9 \mathrm{~m} / \mathrm{s}$. For comparison, the performance of $K_{0}$ at these operating points is shown in Table I.

\section{A. Operation at $15 \mathrm{~m} / \mathrm{s}$}

In this subsection, we consider operation around $v_{\text {nom }}=$ $15 \mathrm{~m} / \mathrm{s}$. We set $p_{\text {nom }}=4 \mathrm{MW}$, which is $1 \mathrm{MW}$ less than what the WT is capable of producing at this wind speed. To attain the power production, we set nominal rotor speed to its rated value, i.e. $\omega_{\mathrm{r}, \text { nom }}=\omega_{\mathrm{r} \text {,rated }}$. At $15 \mathrm{~m} / \mathrm{s}$, this choice of operating point is consistent with the operating points in [18], [25]-[27].

The bounds in Problem $1-2$ are set to $\bar{\omega}_{r}=5.4 \cdot 10^{-3}, \bar{\beta}=$ $0.19, \bar{m}_{g}=200$ and $\bar{p}=12 \cdot 10^{3}$. Note that all the bounds in (3) and (4), except the bound on the generator torque activity, are set according to the performance of $K_{0}^{15}$. The generator torque bound, $\bar{m}_{g}$, is set higher because at $p_{\text {nom }}=$ $4 \mathrm{MW}$, the WT operates well below its rated torque level and can allow more variation in the generator torque.

Next, we compute the solution to Problem 1 and Problem 2 for $\eta \in[0,1]$. Figure 3 shows the complete trade off curve for Problem 1 (solid black) and the leftmost part of the trade off curve for Problem 2 (dashed blue). Figure 3 also shows the trade off curves obtained by solving Problem 1 with different values of $\bar{p}$ (gray). As $\bar{p}$ increases, these trade off curves approach the trade off curve of Problem 2 (dashed blue). Above some level of $\bar{p}$, the leftmost part of the curves coincide with the trade off curve of Problem 2. This implies that trade offs characterized by low shaft loads (large $\eta$ ) can be attained with less power variation than trade offs characterized by low tower loads (small $\eta$ ).

In order to understand how relaxing the power tracking requirement leads to reduced tower loading, we will compare the responses of a Policy 1 controller, denoted $K_{1}^{15}$, and a Policy 2 controller, denoted $K_{2}^{15}$, to the "Mexican hat" gust illustrated in Figure 4. Both $K_{1}^{15}$ and $K_{2}^{15}$ are designed with zero weight on the shaft load (i.e. $\eta=0$ ). The performance of these controllers is shown in Table I. Although they result in high shaft loads, they allow us to study an ideal response in terms of the tower load. The result is shown in Figure 5. 


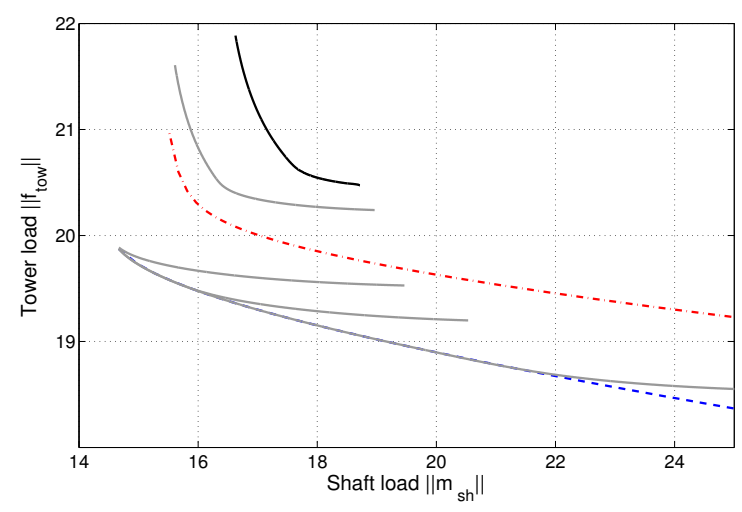

Fig. 3. Trade offs at $v_{\text {nom }}=15 \mathrm{~m} / \mathrm{s}$ between tower and shaft loads under Policy 1 (solid black), Policy 2 (dashed blue) and Policy 3 for $N=2$ WTs (dash-dotted red). The gray curves are trade off curves for Problem 1 with $\bar{p}=\{15,25,30,40\} \mathrm{kW}$. The increase in power fluctuations, $\|p\|$, needed to obtain a simultaneous reduction in tower and shaft loads does not exceed $40 \mathrm{~kW}$.

Under $K_{1}^{15}$, the generator torque tends to decrease whenever the rotor speed increases, thereby accelerating the rotor speed deviations. This behavior is due to the bound on power variations (4) and the algebraic relation between power, generator torque and generator speed in (1). The acceleration caused by the generator torque increases the pitch ef-

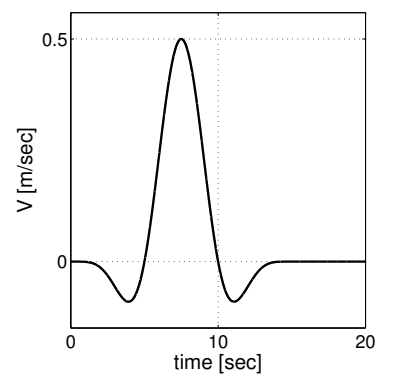

Fig. 4. Mexican hat gust. fort needed to damp rotor speed variations. This behavior is not present in $K_{2}^{15}$ which applies a decelerating torque at the expense of larger power fluctuations. This additional damping unloads the pitch actuator in terms of rotor speed damping and enables a pitch behavior which is better suited with respect to tower loading.

To further illustrate that the possibility to reduce the tower load is linked to the rotor speed constraint, we introduce the Policy 1 controller $K_{1, r}^{15}$ and the Policy 2 controller $K_{2, r}^{15}$. They are designed with $\eta=0, \bar{\omega}_{r}=\infty, \bar{\beta}=$ $0.19, \bar{m}_{g}=200$ and $\bar{p}=12 \cdot 10^{3}$. The difference between these controllers and $K_{1}^{15}$ and $K_{2}^{15}$ is that the constraint on rotor speed has been removed. The performances of $K_{1, r}^{15}$ and $K_{2, r}^{15}$ is shown in Table I. The results show that if there were no need to control the rotor speed, the tower load under Policy 1 would be at level with the tower load under Policy 2. Moreover, the difference between $K_{1, r}^{15}$ and $K_{2, r}^{15}$ shows that removing the power constraint in addition to the rotor speed constraint only results in a minor additional tower load reduction.

Remark 2: Unlike $K_{2}^{15}$ and $K_{1, r}^{15}, K_{2, r}^{15}$ manages to reduce both tower and shaft loads. This will be further explained in the next subsection.
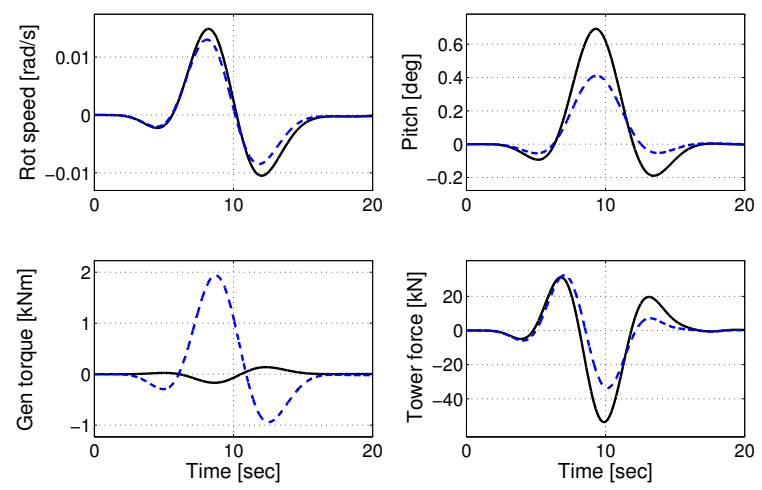

Fig. 5. Response of $K_{1}^{15}$ (solid black) and $K_{2}^{15}$ (dashed blue) to the Mexican hat gust. All plots show deviations from nominal values. The advantage with respect to tower loading of allowing $K_{2}^{15}$ to adjust the power production is that it can use the generator torque to damp rotor speed variations. This additional damping unloads the pitch actuator and enables a pitch behavior that is better suited with respect to tower loading. This behavior is not present in $K_{1}^{15}$ which, in order to track the power demand, has a torque behavior which exacerbates the rotor speed variations.

\section{B. Operation at $9 \mathrm{~m} / \mathrm{s}$}

We now consider operation around $v_{\text {nom }}=9 \mathrm{~m} / \mathrm{s}$ and set the nominal power production to $p_{\text {nom }}=2 \mathrm{MW}$, which is $0.6 \mathrm{MW}$ less than what the WT is capable of producing. This time the nominal power production is attained by setting the nominal rotor speed below rated rotor speed $^{1}, \omega_{\mathrm{r}, \mathrm{nom}}=$ $\frac{\lambda^{*} v_{\text {nom }}}{R}$, where $\lambda^{*}$ is the tip-speed ratio that corresponds to the highest nominal power extraction.

As before, we begin by setting the bounds on the constraints (3) and (4): $\bar{\omega}_{r}=115 \cdot 10^{-3}, \bar{\beta}=0.19, \bar{m}_{g}=200$ and $\bar{p}=12 \cdot 10^{3}$. All bounds, except the bound on rotor speed variations, are equal to those used in Section III-A. The constraint on rotor speed has been relaxed compared to Section III-A because at $9 \mathrm{~m} / \mathrm{s}$ the WT operates well below rated rotor speed. The bound used here is set so that the rotor speed stays below its rated value $95 \%$ of the time.

Figure 6 shows the trade off curve under Policy 1 (solid black) and Policy 2 (blue circle). Under Policy 2 there is no trade off between reducing tower and shaft loads. This is because, as explained in Section III-A, the constraint on the power variations (4) increases the control effort needed in order to limit the rotor speed variations. Because of the high bound on the allowed rotor speed variation at $v_{\text {nom }}=9$ $\mathrm{m} / \mathrm{s}$, removing the power tracking requirement is enough to deactivate the rotor speed constraint. Then, since the generator torque has a relatively small effect on the tower motion, the optimal Policy 2 controller, denoted $K_{2}^{9}$, can be designed in two separate steps. First, a pitch control loop is designed to minimize the tower load. Second, a generator torque loop is designed to minimize the shaft load. The performance of $K_{2}^{9}$ together with a Policy 1 controller, denoted $K_{1}^{9}$, is presented in Table I.

Comparing Figure 6 with Figure 3, we see that both the

\footnotetext{
${ }^{1}$ In general there are several pitch angle and rotor speed configurations that result in the same power production. The configuration used here is consistent with [26], [27].
} 


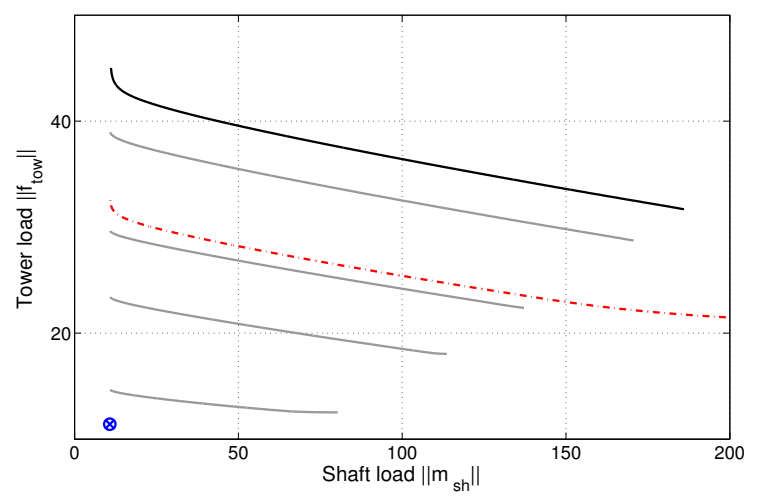

Fig. 6. Trade offs at $v_{\text {nom }}=9 \mathrm{~m} / \mathrm{s}$ between tower and shaft loads for a wind turbine under Policy 1 (solid black), Policy 2 (blue circle), and Policy 3 for $N=2$ WTs (dash-dotted red). The gray curves are trade off curves for Problem 1 with $\bar{p}=\{30,70,100,150\} \mathrm{kW}$. There is no trade off between reducing tower and shaft loads under Policy 2. The Policy 3 curve shows that by coordinating the power of only two WTs it is possible to track a total power demand and retain a significant portion of the load reduction under Policy 2 at the same time.

load reductions and the power variation, $\|p\|$, needed to attain the reductions are larger at $9 \mathrm{~m} / \mathrm{s}$ than at $15 \mathrm{~m} / \mathrm{s}$. This indicates that power coordination might be especially useful at low wind speeds.

Remark 3: The reason for the larger load reductions at $9 \mathrm{~m} / \mathrm{s}$ compared to $15 \mathrm{~m} / \mathrm{s}$ is that, at low wind speeds, the constraint (4) has a larger effect on the rotor speed. Therefore, removing this effect is especially beneficial at low wind speeds.

\section{COORDination}

In Section III we showed that a WT can benefit in terms of tower and shaft loading by allowing larger fluctuations around its power set point. In this section, we demonstrate that when several WTs operate in a WPP, part of this benefit can be retained while jointly tracking a total power demand. The power coordination needed in order to achieve this is referred to as power tracking Policy 3. The controller in this policy is given by the solution to Problem 3 stated below.

Problem 3 (Coordinated power tracking): Consider $N$ WT plants $P_{1}, \ldots, P_{N}$. Given $\eta \in[0,1]$, find stabilizing controllers

$$
K_{3, i}:\left[\begin{array}{c}
y_{1} \\
\vdots \\
y_{N}
\end{array}\right] \rightarrow u_{i}, i=1, \ldots, N
$$

that satisfy (3) locally and minimize

$$
\sum_{i=1}^{N}\left(\eta\left\|m_{\mathrm{sh}, \mathrm{i}}\right\|^{2}+(1-\eta)\left\|f_{\text {tow }, \mathrm{i}}\right\|^{2}\right)
$$

subject to the joint power constraint

$$
\left\|\sum_{i=1}^{N} p_{i}\right\| \leq \bar{p} \sqrt{N}
$$

The bound in (5) corresponds to the joint power variation of $N$ WTs operating under Policy 1 . Indeed, since the power fluctuations from such WTs are assumed to be uncorrelated (see Section II-C), we have

$$
\left\|\sum_{i=1}^{N} p_{i}\right\|^{2}=\sum_{i=1}^{N}\left\|p_{i}\right\|^{2}=N \bar{p}^{2} .
$$

Note that (loosely speaking), Problem 3 is a relaxation of Problem 1, and that Problem 2 is a relaxation of Problem 3. More specifically, let $J_{\text {Policy } 1}$ and $J_{\text {Policy } 2}$ denote the optimal cost in Problem 1 and Problem 2, respectively. Also let $J_{N \text {,Policy } 3}$ be the local cost for a WT in Problem 3. Then, provided that both the bounds on the constraints and $\eta$ in Problems $1-3$ are identical, we have

$$
J_{\text {Policy } 2} \leq J_{N, \text { Policy } 3} \leq J_{\text {Policy } 1}, \text { for } N=1,2, \ldots
$$

Moreover, it can be shown that as $N$ grows large, Policy 3 cost approaches Policy 2 cost. That is,

$$
\lim _{N \rightarrow \infty} J_{N, \text { Policy } 3}=J_{\text {Policy } 2} \text {. }
$$

In particular, this means that Problem 2 provides a tight upper bound on the improvement that can be obtained by coordinating power.

Next, we consider two WTs $(N=2)$, each operating at $v_{\text {nom }}=9 \mathrm{~m} / \mathrm{s}$ with a nominal power production of $p_{\text {nom }}=$ $2 \mathrm{MW}$. We set $\bar{\omega}_{r}=115 \cdot 10^{-3}, \bar{\beta}=0.19, \bar{m}_{g}=200$ and $\bar{p}=12 \cdot 10^{3}$, which is in accordance with the bounds used to design Policy 1 and Policy 2 controllers in Section III-B. The trade off between the two loads in Problem 3 is shown in Figure 6 (red dash-dotted). It shows that by coordinating its power production with one other WT, a WT can retain a significant portion of the load reduction possible under Policy 2. The trade off curve for a WT operating at $v_{\text {nom }}=$ $15 \mathrm{~m} / \mathrm{s}$ and $p_{\text {nom }}=4 \mathrm{MW}$ is constructed analogously and shown in Figure 3.

Let $K_{3,1}$ and $K_{3,2}$ denote the Policy 3 controllers designed to match the shaft load performance of the Policy 1 controller $K_{1}^{9}$. Their performance is listed in Table I. Figure 8 shows the power responses of $K_{3,1}$ and $K_{1}^{9}$ to the turbulent wind in Figure 7. Note that the variation in total plant power is almost identical under both policies (Figure 8 left). However, because $K_{3,1}^{9}$ coordinates its power production with $K_{3,2}^{9}$, it may allow larger fluctuations in its production than $K_{1}^{9}$.

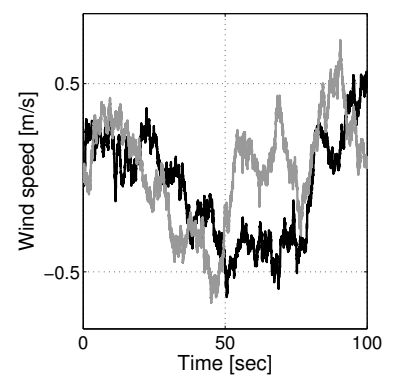

Fig. 7. Turbulent wind speed variations at WT 1 (black) and WT 2 (gray).

\section{Conclusions}

Dynamic power coordination (DPC) allows wind turbines (WTs) in a wind power plant to vary their individual power production as long as the sum of their production meets a total power demand. This paper provides insight into the 

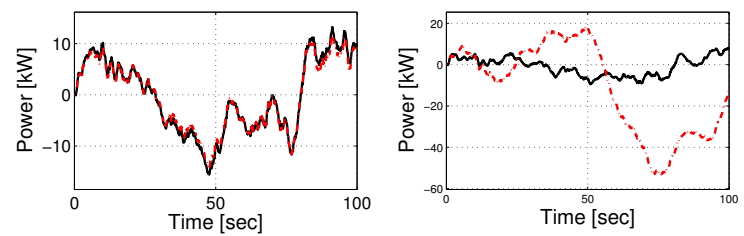

Fig. 8. Power fluctuations in response to turbulent wind speeds in Figure 7 under Policy 1 (solid black) and Policy 3 (dash-dotted red). The left plot illustrates the total power variations of the two wind turbines (the curves overlap almost perfectly) and the right plot shows the local power variations at WT 1.

mechanisms that make DPC useful for reducing fatigue loads on the WT tower and on the low speed shaft.

We saw that a tight constraint on a WT's power production restricts its generator torque behavior and leaves the pitch angle as the only control signal to regulate the rotor speed and the loads. Hence, a benefit of allowing the WT to vary its power is that this restriction is removed. Another benefit is that it helps to reduce rotor speed variations. This effect is especially important because the need to regulate the rotor speed variations causes trade off between reducing tower and shaft loads.

We compared the load reduction that could be obtained by allowing a WT to vary its power at two different nominal wind speeds. At $15 \mathrm{~m} / \mathrm{s}$, where the WT operated at rated rotor speed, there was a trade off between reducing tower and shaft loads. In this case, a reasonable range of trade offs could be attained at the expense of a moderate increase in power variation. At $9 \mathrm{~m} / \mathrm{s}$, where the WT operated below rated rotor speed, the situation was different. In this case, partially due to the relaxed requirements on the rotor speed control, there was no conflict between reducing tower and shaft loads. Also, the load reduction at $9 \mathrm{~m} / \mathrm{s}$ was larger than at $15 \mathrm{~m} / \mathrm{s}$, but came at the expense of considerable power variations. This implies that DPC might be especially beneficial at low wind speeds.

Finally, we showed how power fluctuation at individual WTs may be compensated by coordination. In particular, we demonstrated that even coordination among two WTs may be enough to obtain substantial load reductions.

\section{ACKNOWLEDGMENTS}

This work was supported by the Swedish Research Council through the LCCC Linnaeus Center and by the European commission through the project AEOLUS.

\section{REFERENCES}

[1] T. D. Couture, K. Cory, C. Kreycik, and E. Williams, "A Policymaker s Guide to Feed-in Tariff Policy Design," National Renewable Energy Laboratory, Tech. Rep. July, 2010.

[2] "Integration of Renewable Resources," California ISO, Tech. Rep., 2010.

[3] "Wind Turbines Connected to Grids with Voltages above $100 \mathrm{kV}$," Elkraft Systems and Eltra, Tech. Rep. November, 2004.

[4] "Technical Requirements for the Connection of Generation Facilities to the Hydro-Québec Transmission System," Hydro-Québec, Tech. Rep. May, 2005.

[5] "Wind Farm Transmission Grid Code Provisions: A Direction by the Commission for Energy Regulation," Commission for Energy Regulation, Tech. Rep. July, 2004.
[6] C. Hiroux and M. Saguan, "Large-scale wind power in European electricity markets : Time for revisiting support schemes and market designs?" Energy Policy, vol. 38, no. 7, pp. 3135-3145, 2010.

[7] C. Klessmann, C. Nabe, and K. Burges, "Pros and cons of exposing renewables to electricity market risks A comparison of the market integration approaches in Germany , Spain , and the UK," Energy Policy, vol. 36, no. 10, pp. 3646-3661, 2008

[8] B. Kirby, M. Milligan, and E. Ela, "Providing Minute-to-Minute Regulation from Wind Plants Preprint," in 9th Annual International Workshop on Large-Scale Integration of Wind Power into Power Systems and Transmission Networks for Offshore Wind Power Plants, Quebec, Canada, October 2010.

[9] E. Bitar, R. Rajagopal, P. Khargonekar, K. Poolla, and P. Varaiya, "Bringing Wind Energy to Market," IEEE Transactions on Power System, vol. 27, no. 3, pp. 1225-1235, 2011.

[10] A. Hansen, P. Sorensen, F. Iov, and F. Blaabjerg, "Centralised power control of wind farm with doubly fed induction generators," Renewable Energy, vol. 31, no. 7, pp. 935-951, June 2006.

[11] T. Kaneko, T. Senjyu, A. Yona, M. Datta, T. Funabashi, and C.-H. Kim, "Output Power Coordination Control for Wind Farm in Small Power System," in Intelligent Systems Applications to Power Systems, 2007.

[12] L.-R. Chang-Chien, C.-M. Hung, and Y.-C. Yin, "Dynamic Reserve Allocation for System Contingency by DFIG Wind Farms," IEEE Transactions on Power Systems, vol. 23, no. 2, pp. 729-736, 2008.

[13] R. de Almeida, E. Castronuovo, and J. Lopes, "Optimum generation control in wind parks when carrying out system operator requests,' IEEE Transactions on Power Systems, vol. 21, no. 2, May 2006.

[14] V. Spudić, M. Jelavić, M. Baotić, and N. Perić, "Hierarchical wind farm control for power/load optimization," in Torque 2010 The science of making torque from wind, 2010, pp. $681-692$.

[15] D. Madjidian, K. Mårtensson, and A. Rantzer, "A Distributed Power Coordination Scheme for Fatigue Load Reduction in Wind Farms," in Proceedings of American Control Conference, 2011.

[16] B. Biegel, "Distributed Control of Wind Farm," Master's thesis, Lund University, 2011

[17] M. Kristalny and D. Madjidian, "Decentralized Feedforward Control of Wind Farms : Prospects and Open Problems," in 50th IEEE Conference on Decision and Control and European Control Conference, Orlando, 2011.

[18] J. D. Grunnet, M. Soltani, T. Knudsen, M. Kragelund, and T. Bak, "Aeolus Toolbox for Dynamics Wind Farm Model, Simulation and Control," in European Wind Energy Conference, 2010.

[19] J. Jonkman, S. Butterfield, W. Musial, and G. Scott, "Definition of a 5-MW Reference Wind Turbine for Offshore System Development," National Renewable Energy Laboratory, Tech. Rep. February, 2009.

[20] SimWindFarm Toolbox. Available at http://www.ictaeolus.eu/SimWindFarm/index.html. Last accessed 20/04/2013.

[21] M. Kristalny, D. Madjidian, and T. Knudsen, "On Using Wind Speed Preview to Reduce Wind Turbine Tower Oscillations," IEEE Transactions on Control Systems Technology, to appear.

[22] A. Vigueras-Rodrguez, P. Srensen, A. Viedma, M. Donovan, and E. G. Lzaro, "Spectral coherence model for power fluctuations in a wind farm," Journal of Wind Engineering and Industrial Aerodynamics, vol. 102, no. 0 , pp. $14-21,2012$.

[23] A. Megretsky and S. Treil, "Power Distribution Inequalities in Optimization and Robustness of Uncertain Systems," Journal of Mathematical Systems, Estimation, and Control, vol. 3, no. 3, pp. 301-319, 1993.

[24] V. Yakubovich, "Nonconvex optimization problem: The infinitehorizon linear-quadratic control problem with quadratic constraints," Systems \& Control Letters, vol. 19, no. 1, pp. 13-22, 1992.

[25] L. M. Fernandez, C. A. Garcia, and F. Jurado, "Comparative study on the performance of control systems for doubly fed induction generator (DFIG) wind turbines operating with power regulation,' Energy, vol. 33, pp. 1438-1452, 2008.

[26] H. T. Ma and B. H. Chowdbury, "Working towards frequency regulation with wind plants : combined control approaches," IET Renewable Power Generation, vol. 4, no. 4, pp. 308-316, 2010.

[27] J. L. Rodríguez-amenedo, S. Arnalte, and J. C. Burgos, "Automatic Generation Control of a Wind Farm With Variable Speed Wind Turbines," IEEE Transactions on Energy Conversion, vol. 17, no. 2, pp. 279-284, 2002 